\title{
THE ZERO-DIVISOR GRAPH UNDER A GROUP ACTION IN A COMMUTATIVE RING
}

\author{
JUNCHEOL HAN
}

\begin{abstract}
Let $R$ be a commutative ring with identity, $X$ the set of all nonzero, nonunits of $R$ and $G$ the group of all units of $R$. We will investigate some ring theoretic properties of $R$ by considering $\Gamma(R)$, the zero-divisor graph of $R$, under the regular action on $X$ by $G$ as follows: (1) If $R$ is a ring such that $X$ is a union of a finite number of orbits under the regular action on $X$ by $G$, then there is a vertex of $\Gamma(R)$ which is adjacent to every other vertex in $\Gamma(R)$ if and only if $R$ is a local ring or $R \simeq \mathbb{Z}_{2} \times F$ where $F$ is a field; (2) If $R$ is a local ring such that $X$ is a union of $n$ distinct orbits under the regular action of $G$ on $X$, then all ideals of $R$ consist of $\left\{\{0\}, J, J^{2}, \ldots, J^{n}, R\right\}$ where $J$ is the Jacobson radical of $R$; (3) If $R$ is a ring such that $X$ is a union of a finite number of orbits under the regular action on $X$ by $G$, then the number of all ideals is finite and is greater than equal to the number of orbits.
\end{abstract}

\section{Introduction and basic definitions}

The zero-divisor graph of a commutative ring has been studied extensitively by Akbari, Anderson, Frazier, Lauve, Livinston and Maimani in [1, 2, 3] since its concept had been introduced by Beck in [4]. Recently, zero-divisor graph of a noncommutative ring (resp. a semigroup) has studied by Redmond and Wu (resp. F. DeMeyer and L. Demeyer) in [9, 10, 11] (resp [5]). Zero-divisor graph is very useful to find the algebraic structures and properties of rings. In this paper, the zero-divisor graph of a commutative ring is also studied by considering some group action.

Throughout this paper all rings are assumed to be rings with identity $1 \neq 0$. For a commutative ring $R$, let $Z(R)$ be the set of all zero-divisors of $R$, and $\Gamma(R)$ be the zero-divisor graph of $R$ consisting of all vertices in $Z(R)^{*}=Z(R) \backslash\{0\}$, the set of all nonzero zero-divisors of $R$, and edges $x \longleftrightarrow y$, which means that $x y=0$ for $x, y \in Z(R)^{*}$. In this paper, a loop (i.e., an edge from some vertex to

Received February 3, 2009.

2000 Mathematics Subject Classification. Primary 13H99; Secondary 16E50.

Key words and phrases. zero-divisor graph, regular action, orbit, local ring.

This study was financially supported by the Research Fund Program of Research Institute for Basic Sciences, Pusan National University, Korea, 2008, Project No. RIBS-PNU-20080088000 . 
itself) can be considered an edge in a zero-divisor graph $\Gamma(R)$. Recall that $\Gamma(R)$ is connected if there is a path between any two distinct vertices. For vertices $x$ and $y$ of $\Gamma(R)$, if there exists a path between $x$ and $y$, we will denote $d(x, y)$ by the length of the shortest path between $x$ and $y$, otherwise, $d(x, y)=\infty$. The diameter of $\Gamma(R)$ (denoted by $\operatorname{diam}(\Gamma(R))$ is defined by the supremum of $d(x, y)$ for all distinct vertices $x$ and $y$ in $\Gamma(R)$. In particular, if $x=y$ and $d(x, x)=k \geq 3$, then the path is called the cycle of length $k$. If $\Gamma(R)$ contains a cycle, then the girth of $\Gamma(R)$ (denoted by $g(\Gamma(R))$ ) is defined by the length of the shortest cycle in $\Gamma(R)$, otherwise, $g(\Gamma(R))=\infty$. In [6, Proposition 1.3.2], if $\Gamma(R)$ contains a cycle, then $1+2 \operatorname{diam}(\Gamma(R)) \geq g(\Gamma(R))$. We say that $\Gamma(R)$ is complete if $x y=0$ for any distinct vertices $x, y$ in $\Gamma(R)$. In [3], Anderson and Livingston have shown that for a commutative $\operatorname{ring} R,(1) \Gamma(R)$ is connected and $3 \geq \operatorname{diam}(\Gamma(R)) ;(2)$ there is a vertex of $\Gamma(R)$ which is adjacent to every other vertex in $\Gamma(R)$ if and only if $R \simeq \mathbb{Z}_{2} \times A$ ( $A$ is an integral domain) or $Z(R)$ is an annihilator ideal.

Let $R$ be a ring, $X(R)$ (simply, denoted by $X$ ) the set of all nonzero, nonunits of $R, G(R)$ (simply, denoted by $G$ ) the group of all units of $R$ and $J$, the Jacobson radical of $R$. In this paper, we will consider a group action of $G$ on $X$ given by $((g, x) \longrightarrow g x)$ from $G \times X$ to $X$, called the regular action. If $\phi: G \times X \longrightarrow X$ is the regular action, then for each $x \in X$, we define the orbit of $x$ by $o(x)=\{\phi(g, x): \forall g \in G\}$. Recall that $G$ is transitive on $X$ (or $G$ acts transitively on $X$ ) if there is an $x \in X$ with $o(x)=X$ and the group action on $X$ by $G$ is trivial if $o(x)=\{x\}$ for all $x \in X$. In [7], it has been shown that if $X$ is a union of a finite $n$ number of orbits under the regular action of $G$ on $X$, then (1) $x^{n+1}=0$ for all $x \in J$, and $X$ is the set of all nonzero left zero-divisors of $R$; (2) $R$ is a local ring, $J^{n} \neq(0)$ and $J^{n+1}=(0)$ if and only if there exists $x \in J$ such that $x^{n} \neq(0)$ if and only if $J>J^{2}>\cdots>J^{n-1}>J^{n} \neq(0)$.

For a subset $S$ of $Z(R)^{*}$, we will denote the induced subgraph of $\Gamma(R)$ with vertices in $S$ by $\Gamma_{S}(R)$, that is, $x, y \in S$ are adjacent in $\Gamma_{S}(R)$ if and only if $x$ and $y$ are adjacent in $\Gamma(R)$. In particular, if $R$ is a commutative ring such that $X$ is a union of a finite number of orbits under the regular action of $G$ on $X$, then $X$ is the set of all nonzero zero-divisors of $R$, i.e., $X=Z(R)^{*}$, and so $\Gamma(R)=\Gamma_{X}(R)$. In Section 2, for a commutative ring $R$ such that $X$ is a union of a $n$ orbits under the regular action on $X$ by $G$, we will investigate some ring theoretic properties of $R$ by considering $\Gamma(R)$, the zero-divisor graph of $R$, as follows: (1) if $n=1$, then $\Gamma(R)$ is complete; (2) there is an element $x \in X$ such that $x$ is adjacent to every other vertex in $\Gamma(R)$ if and only if $R$ is a local ring or $R \simeq \mathbb{Z}_{2} \times F$ ( $F$ is a field); (3) if $R$ is a local ring, then every ideal of $R$ is an annihilator of some element $x \in X$ (denoted by ann $(x)$ ); (3) the number of all ideals in $R$ is equal to the number of all annihilators in $R$ and is greater than or equal to $n$, the number of orbits.

Recall that a ring $R$ is called von Neumann regular (simply, regular) (resp. unit-regular) if for every $x \in R$ there exists $y \in R$ (resp. $g \in G$ ) such that $x y x=x$ (resp. $x g x=x$ ). Note that for a commutative ring $R, R$ is regular if 
and only if $R$ is unit-regular. In Section 3, we will investigate some properties of a commutative regular ring $R$ as follows: (1) $\Gamma_{X}(R)$ is complete if and only if the set of all idempotents in $R$ is orthogonal and the regular action of $G$ on $X$ is trivial; (2) if $2=2 \cdot 1$ is a unit in $R$, then there exists a cycle of length 4 in $\Gamma(R)$.

\section{Zero-divisor graph under the regular action}

For each $x \in X$, we will denote the set of every element which is adjacent to $x$ by $a v(x)$. In fact, $a v(x)=\operatorname{ann}(x)^{*}=\operatorname{ann}(x) \backslash\{0\}$.

Proposition 2.1. Let $R$ be a commutative ring. If the regular action of $G$ on $X$ is transitive, then $\Gamma_{X}(R)$ is complete.

Proof. It follows from [7, Theorem 2.2].

Example 1 (See Example 2.1 in [3]). Let $R_{1}=\mathbb{Z}_{9}$ and $R_{2}=\mathbb{Z}_{2} \times \mathbb{Z}_{2}$. Even though $R_{1}$ is not isomorphic to $R_{2}, \Gamma\left(R_{1}\right)=\Gamma\left(R_{2}\right)$. On the other hand, we can note that $(1)$ all the vertices of $\Gamma\left(R_{1}\right)$ are nilpotent but all the vertices of $\Gamma\left(R_{2}\right)$ are not nilpotent; (2) the adjacency matrix of $R_{1}$ is not also equal to the one of $R_{2} ;(3)$ the regular action in $R_{1}$ is transitive but the regular action in $R_{2}$ is trivial.

Example 2 (See Example 2.1 in [3]). Let $R_{1}=\mathbb{Z}_{2}[x, y] /\left\langle x^{2}, x y, y^{2}\right\rangle$ and $R_{2}=$ $F_{4}[x] /\left\langle x^{2}\right\rangle$. Even though $R_{1}$ is not isomorphic to $R_{2}$, but $\Gamma\left(R_{1}\right)=\Gamma\left(R_{2}\right)$. On the other hand, we can note that (1) all the vertices of $\Gamma\left(R_{1}\right)\left(\operatorname{resp} . \Gamma\left(R_{2}\right)\right)$ are nilpotent; (2) the adjacency matrix of $R_{1}$ is equal to the one of $R_{2} ;(3)$ the regular action in $R_{1}$ is trivial but the regular action in $R_{2}$ is transitive.

Proposition 2.2. Let $R$ be a commutative ring such that $X$ is a union of 2 orbits $o(x)$ and $o(y)$ under the regular action on $X$ by $G$. If $\Gamma_{o(x)}(R)$ and $\Gamma_{o(y)}(R)$ are complete, then $\Gamma_{X}(R)$ is complete.

Proof. Note that the set of all the vertices of $\Gamma(R)$ is $X=o(x) \cup o(y)$. Since $\Gamma_{X}(R)$ is connected by [3, Theorem 2.3], there exists $a \in o(x)$ and $b \in o(y)$ such that $a b=0$. Let $x_{1} \in o(x)$ (resp. $y_{1} \in o(y)$ ) be arbitrary. Then $x_{1}=g a$ and $y_{1}=h b$ for some $g, h \in G$, and then $x_{1} y_{1}=(g h)(a b)=0$. Hence $X$ is complete.

Lemma 2.3. Let $R$ be a commutative ring such that $X$ is a union of finite number of orbits under the regular action on $X$ by $G$. Then for each $x \in X$, $a v(x)$ is a union of finite number of orbits.

Proof. It follows from the observation that $a v(x)=\bigcup_{y \in a v(x)} o(y)$ for each $x \in X$.

Theorem 2.4. Let $R$ be a commutative ring such that $X$ is a union of a finite number of orbits under the regular action on $X$ by $G$. Then there is a vertex of $\Gamma(R)$ which is adjacent to every other vertex in $\Gamma(R)$ if and only if $R$ is a local ring or $R \simeq \mathbb{Z}_{2} \times F$ where $F$ is a field. 
Proof. $(\Rightarrow)$ Suppose that there is a vertex of $\Gamma(R)$ which is adjacent to every other vertex in $\Gamma(R)$. Then $R \simeq \mathbb{Z}_{2} \times A$ ( $A$ is an integral domain) or $Z(R)$ is an annihilator ideal by [1, Theorem 2.5]. Let $X$ be a union of $n$ distinct orbits under the regular action on $X$ by $G$. Then $Z(R)^{*}=X$ by [7, Lemma 2.1]. Since $Z(R)^{*}=X$, in case that $R \simeq \mathbb{Z}_{2} \times A, A$ must be a field; in case that $Z(R)$ is an annihilator ideal, $Z(R)=X \cup\{0\}$ is an ideal, which means that $R$ is a local ring.

$(\Leftarrow)$ Suppose that $R$ is a local ring. Then there exists $x \in X$ such that $x^{n} \neq 0=x^{n+1}$ and $X=o(x) \cup o\left(x^{2}\right) \cup \cdots \cup o\left(x^{n}\right)$ by [7, Lemma 2.3]. Thus $a v\left(x^{n}\right)=X$ and so there is a vertex of $\Gamma(R)$ which is adjacent to every other vertex in $\Gamma(R)$. Suppose that $R \simeq \mathbb{Z}_{2} \times F$ where $F$ is a field. Without loss of generality, we can let $R=\mathbb{Z}_{2} \times F$. Then there exists $(1,0) \in R$ such that $a v((1,0))=X$, and so there is a vertex of $\Gamma(R)$ which is adjacent to every other vertex in $\Gamma(R)$.

Corollary 2.5. Let $R$ be a finite commutative ring. Then there is a vertex of $\Gamma(R)$ which is adjacent to every other vertex in $\Gamma(R)$ if and only if $R$ is a local ring or $R \simeq \mathbb{Z}_{2} \times F$ where $F$ is a finite field.

Proof. Since $R$ is a finite commutative ring, clearly $X$ is a union of finite number of orbits under the regular action of $G$ on $X$. Hence it follows from Theorem 2.4 .

Proposition 2.6. Let $R$ be a commutative ring with $X=o(x) \cup o\left(x^{2}\right) \cup \cdots \cup$ $o\left(x^{n}\right)$ under the regular action on $X$ by $G$ for some positive integer $n$. If $n=1$ and $|X| \geq 3$, or $n=2$ and $o\left(x^{2}\right) \neq\left\{x^{2}\right\}$, or $n=3$ and $o\left(x^{i}\right) \neq\left\{x^{i}\right\}$ for some $i=2$ or 3 , or $n \geq 4$, then there exists a cycle of length 3 in $\Gamma(R)$.

Proof. If $n=1$, i.e., the regular action is transitive, then $\Gamma(R)$ is complete by Proposition 2.1. Since $|X| \geq 3$, there exists a cycle of length 3 in $\Gamma(R)$. If $n=2$ and $o\left(x^{2}\right) \neq\left\{x^{2}\right\}$, then there exists $g \in G$ such that $g x^{2} \neq x^{2}$. Since $X=o(x) \cup o\left(x^{2}\right)$ and $x^{2} g \in X, g x^{2}=h x$ or $h x^{2}$ for some $h \in G$. Thus $x^{2} \longrightarrow x \longrightarrow g x^{2} \longrightarrow x^{2}$ is a cycle of length 3 . If $n=3$ and $o_{r}\left(x^{i}\right) \neq\left\{x^{i}\right\}$ for some $i=2$ or 3 , then there exists $g \in G$ such that $g x^{i} \neq x^{i}$. Since $X=o(x) \cup o\left(x^{2}\right) \cup o\left(x^{3}\right)$ and $g x^{i} \in X, g x^{i}=h x$ or $h x^{2}$ or $h x^{3}$ for some $h \in G$. Thus $x^{3} \longrightarrow x^{2} \longrightarrow g x^{i} \longrightarrow x^{3}$ is a cycle of length 3 . Finally, if $n \geq 4$, then clearly $x^{n-2} \longrightarrow x^{n-1} \longrightarrow x^{n} \longrightarrow x^{n-2}$ is a cycle of length 3 .

Theorem 2.7. Let $R$ be a local commutative ring such that $X$ is a union of $n$ distinct orbits under the regular action of $G$ on $X$. Then the set of all the distinct nonzero proper ideals of $R$ consists of $\left\{\{0\}, J, J^{2}, \ldots, J^{n}, R\right\}$.

Proof. Since $R$ is a local ring with identity such that $X$ is a union of $n$ distinct orbits under the regular action of $G$ on $X$, there exists $x \in X$ such that $x^{n} \neq 0=x^{n+1}, X=o(x) \cup o\left(x^{2}\right) \cup \cdots \cup o\left(x^{n}\right)$ by [7, Lemma 2.3] and also $J^{n} \neq\{0\}=J^{n+1}$ by [7, Lemma 2.9]. Thus $J \supset J^{2} \supset \cdots \supset J^{n}$ and $J^{i} \neq J^{j}$ for all $i, j=1, \ldots, n(i \neq j)$. Consider $a v(x), a v\left(x^{2}\right), \ldots, a v\left(x^{n}\right)$. 
Since $x^{n-i+1}, x^{n-i+2}, \ldots, x^{n} \in a v\left(x^{i}\right)$ for all $i=1,2, \ldots, n$, we can have that $a v\left(x^{i}\right)=o\left(x^{n-i+1}\right) \cup o\left(x^{n-i+2}\right) \cup \cdots \cup o\left(x^{n}\right)$. Also we can note that $a v\left(x^{j}\right) \neq a v\left(x^{k}\right)$ and $a v\left(x^{j}\right) \supset a v\left(x^{k}\right)$ for all $j, k(n \geq j>k \geq 1)$. Next, we will show that $J^{k}=\operatorname{ann}\left(x^{n-k+1}\right)\left(=a v\left(x^{n-k+1}\right) \cup\{0\}\right)$ for all $k=1, \ldots, n$ by using induction on $n$. When $n=1, J=\operatorname{ann}(x)$ since $X=a v(x)$. Assume that $J^{k}=\operatorname{ann}\left(x^{n-k+1}\right)$ holds. To show that $J^{k+1}=\operatorname{ann}\left(x^{n-k}\right)$, let $y(\neq 0) \in J^{k+1}$ be arbitrary. Then $y=y_{1} y_{2}$ for some $y_{1} \in J^{k}, y_{2} \in J$. By assumption, $y_{1} \in \operatorname{ann}\left(x^{n-k+1}\right)$ and $y_{2} \in \operatorname{ann}\left(x^{n}\right)$. Since $\operatorname{ann}\left(x^{n-k+1}\right) \backslash\{0\}=$ $a v\left(x^{n-k+1}\right)=o\left(x^{k}\right) \cup o\left(x^{k+1}\right) \cup \cdots \cup o\left(x^{n}\right)$ and $\operatorname{ann}\left(x^{n}\right) \backslash\{0\}=a v\left(x^{n}\right)=$ $o(x) \cup o\left(x^{2}\right) \cup \cdots \cup o\left(x^{n}\right), y_{1}=a x^{k}, y_{2}=b x$ for some $a, b \in R \backslash\{0\}$. Thus $y x^{n-k}=y_{1} y_{2} x^{n-k}=a b x^{k+1} x^{n-k}=a b x^{n+1}=0$, which implies $y \in a v\left(x^{n-k}\right)$. Hence $J^{k+1} \subset \operatorname{ann}\left(x^{n-k}\right)$. To show the convere inclusion holds, let $z \in$ $\operatorname{ann}\left(x^{n-k}\right)$ be arbitrary. Then $z x^{n-k}=0$. Since ann $\left(x^{n-k}\right) \backslash\{0\}=\operatorname{av}\left(x^{n-k}\right)=$ $o\left(x^{k+1}\right) \cup o\left(x^{k+2}\right) \cup \cdots \cup o\left(x^{n}\right), z \in o\left(x^{i}\right)$ for some $i(n \geq i \geq k+1)$, and so $z=g x^{i}$ for some $g \in G$. Thus $z=g x^{i}=(g x)\left(x^{i-1}\right) \in J^{k+1}$ since $g x \in J$ and $x^{i-1} \in J^{k}$. Thus $J^{k+1} \supset \operatorname{ann}\left(x^{n-k}\right)$. Hence we have $J^{k+1}=\operatorname{ann}\left(x^{n-k}\right)$. Let $A=\left\{J, J^{2}, \ldots, J^{n}\right\}$. Therefore, $J^{k}=\operatorname{ann}\left(x^{n-k+1}\right)\left(=\operatorname{av}\left(x^{n-k+1}\right) \cup\{0\}\right)$ for all $k=1, \ldots, n$. Finally, we will show that for any nonzero proper ideal $I$ of $R, I \in A$. Since $I$ is a nonzero ideal of $R$, there exists $y \in X$. Since $X=o(x) \cup o\left(x^{2}\right) \cup \cdots \cup o\left(x^{n}\right), y \in o\left(x^{i}\right)$ for some $i$, and then $o\left(x^{i}\right) \subset I$. Since $x^{i} \in I$ and $I$ is an ideal of $R, x^{i+1}, \ldots, x^{n} \in I$, and so $o\left(x^{i}\right), \ldots, o\left(x^{n}\right) \subset I$, which implies that $J^{i}=o\left(x^{i}\right) \cup \cdots \cup o\left(x^{n}\right) \cup\{0\} \subseteq I$. If $I \neq J^{i}$, then there exists $z \in I \backslash J^{i}$. Then $z \in o\left(x^{j}\right)$ for some $j(i>j \geq 1)$. By the same argument given as above, $J^{j} \subseteq I(i>j)$. If $I \neq J^{j}$, then we will continue in this way. Since $A=\left\{J, J^{2}, \ldots, J^{n}\right\}$ is a finite set of ideals in $R, I$ must be $J^{k}$ for some $k(n \geq k \geq 1)$. Hence the set of all ideals of $R$ consists of $\left\{\{0\}, J, J^{2}, \ldots, J^{n}, R\right\}$.

For any set $S$, we denote the cardinality of $S$ by $|S|$.

Corollary 2.8. Let $R$ be a local commutative ring such that $X$ is a union of $n$ orbits under the regular action of $G$ on $X$. If $S=\{a v(a): \forall a \in X\}$, then $S=\left\{J^{i} \backslash\{0\}: i=1, \ldots, n\right\}$, and so $|S|=n$.

Proof. Let $I_{a}^{*}=a v(a)$ for all $a \in X$. Then $I_{a}^{*}$ is a union of some orbits by Lemma 2.3. Since $I_{a}=I_{a}^{*} \cup\{0\}=\operatorname{ann}(a)$ is an ideal of $R, I_{a}=J^{k}$ for some $k$ $(n \geq k \geq 1)$ by Theorem 2.7. In the proof in Theorem 2.7, $J^{k}=\operatorname{ann}\left(x^{n-k+1}\right)$. Hence we have the result from Theorem 2.7.

Corollary 2.9. Let $R$ be a finite local commutative ring such that $X$ is a union of $n$ orbits under the regular action of $G$ on $X$ and let $m$ be the number of all ideals of $R$. Then

$$
m-2=n=\frac{1}{|G|} \sum_{g \in G}\left|X_{g}\right|,
$$

where $X_{g}=\{x \in X: g x=x\}$. 
Proof. It follows from the Theorem 2.7 and the Burnside's formula.

Lemma 2.10. Let $R=R_{1} \times R_{2} \times \cdots \times R_{t}$ be the direct product of commutative rings $R_{1}, R_{2}, \ldots, R_{t}$ and let $B=\{\operatorname{ann}(x): \forall x \in X\} \cup\{\{0\}, R\}$ and $B_{i}=$ $\left\{\operatorname{ann}\left(x_{i}\right): \forall x \in X\right\} \cup\left\{\left\{0_{i}\right\}, R_{i}\right\}$ for all $i=1, \ldots, t$ where each $X_{i}$ is the set of all nonzero, nonunits of $R_{i}$ and $0_{i}$ is the additive identity of $R_{i}$. Then $B_{1} \times B_{2} \times \cdots \times B_{t} \subseteq B$

Proof. Let $b_{1} \times b_{2} \times \cdots \times b_{t} \in B_{1} \times B_{2} \times \cdots \times B_{t}$ be arbitrary.

Case 1. $b_{i} \neq\left\{0_{i}\right\}, R_{i}$ for all $i$, i.e., $b_{i}=\operatorname{ann}\left(x_{i}\right)$ for some $x_{i} \in X_{i}$.

Thus $b_{1} \times b_{2} \times \cdots \times b_{t}=\operatorname{ann}\left(x_{1}\right) \times \operatorname{ann}\left(x_{2}\right) \times \cdots \times \operatorname{ann}\left(x_{t}\right)$. Then clearly, $\operatorname{ann}\left(x_{1}\right) \times \operatorname{ann}\left(x_{2}\right) \times \cdots \times \operatorname{ann}\left(x_{t}\right)=\operatorname{ann}\left(\left(x_{1}, x_{2}, \ldots, x_{t}\right)\right) \in B$.

Case 2. $b_{i}=\left\{0_{i}\right\}$ for some $i$.

Thus $b_{1} \times b_{2} \times \cdots \times b_{t}=\operatorname{ann}\left(x_{1}\right) \times \cdots \times\left\{0_{i}\right\} \times \cdots \times \operatorname{ann}\left(x_{t}\right)$. Then $\operatorname{ann}\left(x_{1}\right) \times \cdots \times\left\{0_{i}\right\} \times \cdots \times \operatorname{ann}\left(x_{t}\right) \subseteq \operatorname{ann}\left(\left(x_{1}, \ldots, 1_{i}, \ldots, x_{t}\right)\right) \in B$, where $1_{i}$ is the unity of $R_{i}$.

Case 3. $b_{i}=R_{i}$ for some $i$.

Thus $b_{1} \times b_{2} \times \cdots \times b_{t}=\operatorname{ann}\left(x_{1}\right) \times \cdots \times R_{i} \times \cdots \times \operatorname{ann}\left(x_{t}\right)$. Then $\operatorname{ann}\left(x_{1}\right) \times$ $\cdots \times R_{i} \times \cdots \times \operatorname{ann}\left(x_{t}\right) \subseteq \operatorname{ann}\left(\left(x_{1}, \ldots, 0_{i}, \ldots, x_{t}\right)\right) \in B$.

Case 4. $b_{i}=\left\{0_{i}\right\}$ for some $i$ and $b_{j}=R_{j}$ for some $j(i \neq j)$.

Thus by Case 2 and Case $3, b_{1} \times \cdots \times b_{i} \times \cdots \times b_{i} \times \cdots \times b_{t}=\operatorname{ann}\left(x_{1}\right) \times$ $\cdots \times\left\{0_{i}\right\} \times \cdots \times R_{j} \times \cdots \times \operatorname{ann}\left(x_{t}\right)$. Then $\operatorname{ann}\left(x_{1}\right) \times \cdots \times\left\{0_{i}\right\} \times \cdots \times R_{j} \times$ $\left.\cdots \times \operatorname{ann}\left(x_{t}\right)\right) \subseteq \operatorname{ann}\left(\left(x_{1}, \ldots, 1_{i}, \ldots, 0_{j}, \ldots, x_{t}\right)\right) \in B$.

Case 5. $b_{i}=\left\{0_{i}\right\}$ or $b_{i}=R_{i}$ for all $i$.

Thus $b_{1} \times \cdots \times b_{i} \times \cdots \times b_{t}=\operatorname{ann}\left(\left(a_{1}, \ldots, \ldots, a_{i}, \ldots, a_{t}\right)\right) \in B$, where $a_{i}=1_{i}$ or $a_{i}=0_{i}$ for all $i$.

Lemma 2.11. Let $R=R_{1} \times R_{2} \times \cdots \times R_{t}$ be the direct product of commutative rings $R_{1}, R_{2}, \ldots, R_{t}$ and let $C=\{o(x): \forall x \in X\} \cup\{\{0\}, R\}$ and $C_{i}=\left\{o\left(x_{i}\right)\right.$ : $\forall x \in X\} \cup\left\{\left\{0_{i}\right\}, R_{i}\right\}$ for all $i=1, \ldots, t$ where each $X_{i}$ is the set of all nonzero, nonunits of $R_{i}$ and $0_{i}$ is the additive identity of $R_{i}$. Then $C \subseteq C_{1} \times C_{2} \times \cdots \times C_{t}$.

Proof. Let $c \in C$ be arbitrary

Case 1. $c=\{0\}$ or $c=R$.

Then clearly, $c \in C_{1} \times C_{2} \times \cdots \times C_{t}$.

Case 2. $c=o(x)$ for some $x=\left(x_{1}, \ldots, x_{t}\right) \in X$.

Subcase 1. $x_{i} \in X_{i}$ for all $i$.

Subcase 2. $x_{i}=0_{i}$ for some $i$.

Then $c=o(x)=o\left(\left(x_{1}, \ldots, 0_{i}, \ldots, x_{t}\right)\right) \subseteq o\left(x_{1}\right) \times \cdots \times\left\{0_{i}\right\} \times \cdots \times o\left(x_{t}\right) \in$ $C_{1} \times \cdots \times C_{i} \times \cdots \times C_{t}$.

Subcase 3. $x_{i}=1_{i}$ for some $i$.

Then $c=o(x)=o\left(\left(x_{1}, \ldots, 1_{i}, \ldots, x_{t}\right)\right) \subseteq o\left(x_{1}\right) \times \cdots \times R_{i} \times \cdots \times o\left(x_{t}\right) \in$ $C_{1} \times \cdots \times C_{i} \times \cdots \times C_{t}$.

Subcase 4. $x_{i}=0_{i}$ for some $i$ and $x_{j}=1_{j}$ for some $j(i \neq j)$.

Thus by Subcase 2 and Subcase $3, c=o(x)=o\left(\left(x_{1}, \ldots, x_{i}, \ldots, x_{t}\right)\right) \subseteq$ $o\left(x_{1}\right) \times \cdots \times\left\{0_{i}\right\} \times \cdots \times R_{j} \times \cdots \times o\left(x_{t}\right) \in C_{1} \times \cdots \times C_{i} \times \cdots \times C_{j} \times \cdots \times C_{t}$. 
Remark 1. Let $R$ be a commutative ring such that $X$ is a union of a finite number of orbits under the regular action of $G$ on $X$. Then $R$ is an Artinian ring since $I \backslash\{0\}$ is a union of some orbits for every ideal $I$ of $R$ by Lemma 2.3. Therefore, $R$ is a finite direct product of Artinian local rings, say $R=$ $R_{1} \times R_{2} \times \cdots \times R_{t}$ with each $R_{i}$ Artinian local $\operatorname{ring}(i=1, \ldots, n)$.

Theorem 2.12. Let $R$ be a commutative ring such that $X$ is a union of $a$ finite number of orbits under the regular action of $G$ on $X$ and let $R=R_{1} \times$ $R_{2} \times \cdots \times R_{t}$ where each $R_{i}$ is Artinian local ring $(i=1, \ldots, n)$ as mentioned in Remark 1. Then

(1) for all ideal $I$ of $R, I=I_{1} \times I_{2} \times \cdots \times I_{t}$ where $I_{i} \in\left\{\left\{0_{i}\right\}, J_{i}, J_{i}^{2}, \ldots, J_{i}^{n_{i}}\right.$, $\left.R_{i}\right\} \quad\left(\left\{0_{i}\right\}\right.$ is the zero ideal of $R_{i}$ and $J_{i}$ is the Jacobson radical of $R_{i}$ with $J_{i}^{n_{i}} \neq\left\{0_{i}\right\}=J_{i}^{n_{i+1}}$ ) for all $i=1, \ldots, t$.

(2) the number of all nonzero proper ideals of $R$ is $\left(n_{1}+2\right) \cdots\left(n_{t}+2\right)-2$, is equal to $|\{a v(x): \forall x \in X\}|$ and greater than or equal to $\mid\{o(x)$ : $\forall x \in X\} \mid$.

Proof. (1) Note that any ideal $I$ of $R$ is of the form $I_{1} \times I_{2} \times \cdots \times I_{t}$ where $I_{i}$ is an ideal of $R_{i}$ for all $i=1, \ldots, n$. Since $R_{i}$ is a local commutative ring for all $i=1, \ldots, n, I_{i} \in\left\{\left\{0_{i}\right\}, J_{i}, J_{i}^{2}, \ldots, J_{i}^{n_{i}}, R_{i}\right\}$ by Theorem 2.7 and so we have the result.

(2) Let $A$ (resp. $A_{i}$ ) be the set of all ideals of $R$ (resp. the set of all ideals of $R_{i}$ ) for all $i 1, \ldots, t, B=\{\operatorname{ann}(x): \forall x \in X\} \cup\{\{0\}, R\}$ and $C=\{o(x)$ : $\forall x \in X\} \cup\{\{0\}, R\}$. By (1), $A=A_{1} \times \cdots \times A_{t}$ and so $|A|=\prod_{i=1}^{t}\left|A_{i}\right|=$ $\left(n_{1}+2\right) \cdots\left(n_{t}+2\right)$. In the proof of Theorem 2.7 , we have that

$$
A_{i}=\left\{\left\{0_{i}\right\}, J_{i}, \ldots, J_{i}^{n_{i}}, R_{i}\right\}
$$

with $J_{i}^{n_{i+1}}=\left\{o_{i}\right\}$ and $J_{i}^{k_{i}}=\operatorname{ann}\left(x_{i}^{n_{i}-k_{i}+1}\right)$ for some $x_{i} \in X_{i}$, the set of all nonzero, nonunits of $R_{i}$ for all $i=1, \ldots, t$ where $n_{i} \geq k_{i} \geq 1$. Since for all $x \in X, \operatorname{ann}(x)$ is a nonzero proper ideal of $R, B \subseteq A$, and so $(|A|-2) \geq$ $|\{\operatorname{ann}(x): \forall x \in X\}|$. Let $B_{i}=\left\{\operatorname{ann}\left(x_{i}\right): \forall x_{i} \in X\right\} \cup\left\{\left\{0_{i}\right\}, R_{i}\right\}$ for all $i=1, \ldots, t$. Clearly, $A_{i} \subseteq B_{i}$ for all $i=1, \ldots, t$. By above $(*)$, we have $B_{i} \subseteq A_{i}$ for all $i=1, \ldots, t$. Therefore, $A_{i}=B_{i}$ for all $i=1, \ldots, t$. By Lemma 2.10, we have $B_{1} \times \cdots \times B_{t} \subseteq B$. Hence $B \subseteq A=A_{1} \times \cdots \times A_{t}=B_{1} \times \cdots \times B_{t}=B$, and so $A=B$. Therefore, $|A|-2=|\{\operatorname{ann}(x): \forall x \in X\}|=|\{a v(x): \forall x \in X\}|$. On the other hand, let $C_{i}=\left\{o\left(y_{i}\right): \forall y_{i} \in X\right\} \cup\left\{\left\{0_{i}\right\}, R_{i}\right\}$ for all $i=1, \ldots, t$. By Lemma 2.11, we also have $C \subseteq C_{1} \times \cdots \times C_{t}$, and so $|C| \leq\left|C_{1}\right| \times \cdots \times\left|C_{t}\right|$. Since $\left|B_{i}\right|=\left|\left\{a v\left(y_{i}\right): \forall y_{i} \in X\right\} \cup\left\{\left\{0_{i}\right\}, R_{i}\right\}\right|=\left|C_{i}\right|$ for all $i=1, \ldots, t$ by Corollary 2.8, $|A|=|B|=\left|B_{1}\right| \times \cdots \times\left|B_{t}\right|=\left|C_{1}\right| \times \cdots \times\left|C_{t}\right| \geq|C|$.

We can have the following question:

Question 1. Let $R$ be a commutative ring with identity such that $X$ is a union of $n$ orbits under the regular action of $G$ on $X$. Is $|\{a v(x): \forall x \in X\}|=$ $|\{o(x): \forall x \in X\}| ?$ 
Example 3. Let $R=\mathbb{Z}_{36}$. Then $R$ has 7 nonzero proper ideals. We can compute that $a v(x)$ and $o(x)$ for all $x \in X$ as follows: $a v(2)=18 R=$ $\{18\}, a v(3)=12 R=\{12,24\}, a v(4)=9 R=\{9,18,27\}, a v(6)=6 R=$ $\{6,12,18,24,30\}, a v(9)=4 R=\{4,8, \ldots, 32\}, a v(12)=3 R=\{3,6, \ldots, 33\}$, $a v(2)=2 R=\{2,4, \ldots, 34\} ; o(18)=\{18\}, o(6)=\{6,30\}, o(9)=\{9,27\}$, $o(12)=\{12,24\}, o(3)=\{3,15,21,33\}, o(2)=\{2,10,14,22,26,34\}$ and $o(4)=$ $\{4,8,16,20,28,32\}$. Note that the number of $a v(x) \mathrm{s}$ ' is 7 and is equal to the number of $o(x) \mathrm{s}^{\prime}$.

Example 4. Let $R=\mathbb{Z}_{3}[x] /\left\langle x^{3}\right\rangle$ and for simple notation, denote $f(x)=$ $f(x)+\left\langle x^{3}\right\rangle \in R$ for all $f(x) \in \mathbb{Z}_{3}[x]$. Then $X=\left\{x, 2 x, x^{2}, 2 x^{2}, x+x^{2}, 2 x+\right.$ $\left.x^{2}, x+2 x^{2}, 2 x+2 x^{2}\right\}$ and $R$ has 2 nonzero proper ideals $x R$ and $x^{2} R$. We can also compute that $a v(y)$ and $o(y)$ for all $y \in X$ as follows: $a v(x)=\left\{x^{2}, 2 x^{2}\right\}$, $a v\left(x^{2}\right)=\left\{x, 2 x, x^{2}, 2 x^{2}, x+x^{2}, 2 x+x^{2}, x+2 x^{2}, 2 x+2 x^{2}\right\} ; o\left(x^{2}\right)=\left\{x^{2}, 2 x^{2}\right\}$, $o(x)=\left\{x, 2 x, x+x^{2}, 2 x+x^{2}, x+2 x^{2}, 2 x+2 x^{2}\right\}$. Note that the number of $a v(y) \mathrm{s}^{\prime}$ is 2 and is also equal to the number of $o(y) \mathrm{s}^{\prime}$.

\section{Zero-divisor graph of regular rings}

In [4], it has been shown that if $R$ is a unit-regular ring, then for every orbit $o(x)(x \in X)$ under the regular action of $G$ on $X$, there exists some idempotent $e \in X$ such that $o(x)=o(e)$. Note that for a commutative ring $R$ with identity, $R$ is regular if and only $R$ is unit-regular.

Proposition 3.1. Let $R$ be a commutative regular ring. Then $\Gamma_{X}(R)$ is complete if and only if the set of all idempotents in $R$ is orthogonal and the regular action of $G$ on $X$ is trivial, i.e., o $(x)=\{x\}$ for all $x \in X$.

Proof. $(\Rightarrow)$ Suppose that $\Gamma_{X}(R)$ is complete. Clearly, the set of all idempotents in $R$ is orthogonal. Assume that the regular action of $G$ on $X$ is not trivial. Then there exists $y \in X$ such that $o(y) \neq\{y\}$. By [8, Lemma 2.3], there exists idempotent $e(\neq y) \in X$ such that $y=g e$ for some $g \in G$. Since $\Gamma_{X}(R)$ is complete and $y, e \in X, 0=y e=(g e) e=g e=y$, a contradiction. Hence the regular action of $G$ on $X$ is trivial.

$(\Leftarrow)$ Suppose that the set of all idempotents in $R$ is orthogonal and the regular action of $G$ on $X$ is trivial. Let $x, y(x \neq y) \in X$ be arbitrary. By [8, Lemma 2.3], there exist idempotents $e_{1}, e_{2} \in X$ such that $o(x)=o\left(e_{1}\right)$ and $o(y)=o\left(e_{2}\right)$. Since the regular action of $G$ on $X$ is trivial, $\{x\}=o(x)=o\left(e_{1}\right)=$ $\left\{e_{1}\right\}$ and $\{y\}=o(y)=o\left(e_{2}\right)=\left\{e_{2}\right\}$, and so $x=e_{1}, y=e_{2}$. Since $x \neq y$, $e_{1} \neq e_{2}$ and so $x y=e_{1} e_{2}=0$ by assumption. Thus $\Gamma_{X}(R)$ is complete.

Lemma 3.2. Let $R$ be a commutative regular ring. Then the following are equivalent:

(1) $x^{2}=x$ for all $x \in X$

(2) the regular action of $G$ on $X$ is trivial;

(3) $G=\{1\}$. 
Proof. $(1) \Rightarrow(2)$. Suppose that $x^{2}=x$ for all $x \in X$. Let $y \in o(x)$ be arbitrary. Then $y=g x$ for some $g \in G$. Since $y \in X, y^{2}=y$ by assumption, and then $y^{2}=(g x)^{2}=g^{2} x=y=g x$, which implies $y=g x=x$, and so $o(x)=\{x\}$. Thus the regular action of $G$ on $X$ is trivial.

$(2) \Rightarrow(3)$. Suppose that the regular action of $G$ on $X$ is trivial and let $e \in X$ be an idempotent. Then $o(e)=\{e\}$ and $o(1-e)=\{1-e\}$, and so $g e=e$ and $g(1-e)=1-e$ for all $g \in G$. Thus $g-e=g(1-e)=1-e$, which implies $g=1$. Thus $G=\{1\}$.

$(3) \Rightarrow(1)$. Suppose that $G=\{1\}$. Let $x \in X$ be arbitrary. Since $G=\{1\}$, $o(x)=\{x\}$, and so $o(x)=\{x\}=e$ for some idempotent $e \in X$ by [8, Lemma 2.3]. Hence $x^{2}=x$ for all $x \in X$.

Corollary 3.3. Let $R$ be a commutative regular ring. Then $\Gamma_{X}(R)$ is complete if and only if the set of all idempotents in $R$ is orthogonal and one of the statements in Lemma 3.2 is satisfied.

Proof. It follows from Proposition 3.1 and Lemma 3.2.

Remark 2. Let $R$ be a ring. If the regular action of $G$ on $X$ is transitive, then there exists no idempotent in $X$. Indeed, assume that there exists an idempotent $e \in X$. Since the regular action of $G$ on $X$ is transitive, $X=$ $o(1-e)$, and then $e=g(1-e)$ for some $g \in G$. Thus $0=e(1-e)=$ $g(1-e)^{2}=g(1-e)$, and so $1=e$, a contradiction. Therefore for a unit-regular (commutative regular) ring $R$ with identity, there is no transitive regular action of $G$ on $X$ by the above argument and [8, Lemma 2.3].

Proposition 3.4. Let $R$ be a commutative regular ring with $X \neq \emptyset$. Then for each $x \in X$, there exists an idempotent $e \in X$ such that av $(x)=a v(e)$.

Proof. By [8, Lemma 2.3], for each $x \in X$ there exists an idempotent $e \in X$ such that $o(x)=0(e)$. Thus $e=g x$ for some $g \in G$, and then $a v(e)=$ $a v(x)$.

Proposition 3.5. Let $R$ be a commutative regular ring such that $2=2 \cdot 1$ is a unit in $R$. Then there exists a cycle of length 4 in $\Gamma(R)$.

Proof. Let $e \in X$ be an idempotent. Since $2=2 \cdot 1 \in G, e \neq 1-e,-e$. Thus $e \longleftrightarrow 1-e \longleftrightarrow-e \longleftrightarrow e-1 \longleftrightarrow e$ is a cycle of length 4 in $\Gamma(R)$.

We note that for any idempotent $e(\neq 0,1)$ in a commutative regular ring $R$, under the regular action of $G$ on $X, o(1-e) \subseteq a v(e)$. In particular, if $R=F_{1} \times F_{2}\left(F_{1}, F_{2}:\right.$ fields $)$, then $o(1-e)=a v(e)$ for all idempotent $e(\neq 0,1) \in R$.

We raise the following question:

Question 2. For any idempotent $e(\neq 0,1)$ in a commutative regular ring $R$ with identity, when is $o(1-e)=a v(e)$ ? 
Acknowledgements. The author thanks the referee for his/her helpful comments for the improvement of the paper, also the author thanks Prof. J. Park at Pusan National University for reading this paper and for kind comments.

\section{References}

[1] S. Akbari and A. Mohammadian, On the zero-divisor graph of a commutative ring, J. Algebra 274 (2004), no. 2, 847-855.

[2] D. F. Anderson, A. Frazier, A. Lauve, and P. S. Livingston, The zero-divisor graph of a commutative ring. II, Ideal theoretic methods in commutative algebra (Columbia, MO, 1999), 61-72, Lecture Notes in Pure and Appl. Math., 220, Dekker, New York, 2001.

[3] D. F. Anderson and P. S. Livingston, The zero-divisor graph of a commutative ring, J. Algebra 217 (1999), no. 2, 434-447.

[4] I. Beck, Coloring of commutative rings, J. Algebra 116 (1988), no. 1, 208-226.

[5] F. DeMeyer and L. DeMeyer, Zero divisor graphs of semigroups, J. Algebra 283 (2005), no. $1,190-198$.

[6] R. Diestel, Graph Theory, pringer-Verlag, New York, 1997.

[7] J. Han, Regular action in a ring with a finite number of orbits, Comm. Algebra 25 (1997), no. 7, 2227-2236.

[8] _ Group actions in a unit-regular ring, Comm. Algebra 27 (1999), no. 7, 33533361.

[9] S. P. Redmond, The zero-divisor graph of non-commutative ring, Internat. J. Commutative Rings 1 (2002), no. 4, 203-211.

[10] - Structure in the zero-divisor graph of a noncommutative ring, Houston J. Math. 30 (2004), no. 2, 345-355.

[11] T. Wu, On directed zero-divisor graphs of finite rings, Discrete Math. 296 (2005), no. $1,73-86$.

Department of Mathematics Education

Pusan National University

PUSAN 609-735, Korea

E-mail address: jchan@pusan.ac.kr 